\title{
Polaritons in modified antiferromagnets
}

\author{
T. L. Fonseca, A. S. Carriço, ${ }^{*}$ and N. S. Almeida \\ Departamento de Física Teórica e Experimental, Centro de Ciências Exatas, \\ Universidade Federal do Rio Grande do Norte, 59072, Natal, Rio Grande do Norte, Brazil
}

(Received 20 September 1991; revised manuscript received 6 April 1992)

\begin{abstract}
A theoretical study of mixed modes of uniaxial Heisenberg antiferromagnets by a dc field is presented. The static field is applied in an arbitrary direction relative to the easy axis and special attention is given to the magnetostatic limit where previous calculations show intriguing behavior of surface modes. Retarded polaritons are also considered and the spectrum that should be observed through the attenuatedtotal-reflection technique is calculated. Numerical results are presented for $\mathrm{MnF}_{2}$ and $\mathrm{FeF}_{2}$ semi-infinite specimens.
\end{abstract}

\section{INTRODUCTION}

Electromagnetic waves coupled to fundamental excitations of materials have been an exhaustively studied subject. $^{1-10}$ In the last few years, special attention has been given to the properties of these composite waves in magnetic materials. It has been shown (see Ref. 11 for a recent review) that bulk and surface polaritons in these materials frequently have intricate and fascinating physical characteristics. Unfortunately, for most materials, experimental checks of theoretical predictions are not available. Usually the theoretical models are too simple to represent real specimens, while real samples are "dirty" enough to be described by any reliable theoretical model. Very frequently, if a more realistic model is used, the results become complex enough to have a clear physical interpretation. On the other hand, neglecting the leading effects may lead to misleading theoretical predictions. The ideal system is one in which the leading physical features are well represented by a simple theoretical model.

Antiferromagnetic materials $\left(\mathrm{FeF}_{2}\right.$ and $\left.\mathrm{MnF}_{2}\right)$ have recently been grown with exceptional good quality. ${ }^{12}$ They are also well described theoretically by the "twosublattice model."4 The main feature of this simple model is that it allows a clear and analytical analysis of the main properties. The reason for this simple and real description of these materials comes from the fact that they have a highly symmetrical structure (bodycentered-tetragonal lattice) which can be seen as two interpenetrating sublattices with a uniaxial anisotropy. In the magnetically ordered state, the magnetic moment of each sublattice has a ferromagnetic order, with the spins of one sublattice parallel to the spins of the other one but in the opposite direction. The exchange interaction, i.e., the effect of one sublattice on the magnetic moment of the other, is the mechanism responsible for keeping the spins of the two sublattices antiparallel. This effect is described by the exchange field $H_{e}$ which, in the longwavelength limit, represents the action of the first sublattice on the second. On the other hand, the effect of anisotropy is taken into account by assuming each magnetic moment in the presence of a dc field $H_{a}$ which pins the spins on the $c$ axis. Using this description it is well known that antiferromagnets, in the absence of a dc external magnetic field, have a resonance frequency ${ }^{2}$ given by $\Omega_{r}=\gamma\left(2 H_{e} H_{a}+H_{a}^{2}\right)^{1 / 2}$, with $\gamma$ denoting the gyromagnetic ratio. It is also verified that for frequencies close to $\Omega_{r}$ the response of the system shows a strong variation and, as a consequence, the specimen becomes optically active. Typical values for this resonance frequency are from $250 \mathrm{GHz}$ to $1 \mathrm{THz}$ (infrared region) if no dc external magnetic field is present.

The near totality of the studies developed for these materials explores the presence of an external static magnetic field applied parallel to the anisotropy axis. It is found that many properties are strongly dependent on the strength of the applied static field, even for fields well below the spin-flop threshold. ${ }^{8,9,11}$ One of these effects is the nonreciprocal propagation of surface waves, 9,11 i.e., surface waves which have different frequencies if either the direction of the applied field or the direction of propagation is reversed. This fascinating feature can be understood through the symmetry arguments given many years ago by Scott and Mills. ${ }^{5}$

Recently the effect of nonsymmetry induced by an external dc magnetic field was the object of an investigation by Almeida and Mills. ${ }^{13-15}$ As usual, the first step was to investigate the effect of the new spin configuration on the dynamic response of these systems. It was shown by these authors that, in the magnetostatic limit and for a dc external field with strength close to $H_{a}$, surface polaritons appear with amazing properties. One remarkable feature of these modes, as shown in Fig. 3 of Ref. 13, is the unresolved crossing of the surface modes which have the propagation direction around $45^{\circ}$ from the easy axis.

The aim of this work is to extend the calculation developed in Ref. 13 to study the retarded modes as well as to investigate the influence of the nonsymmetry induced by a dc external magnetic field, applied in the oblique direction relative to the easy axis, on the magnetostatic modes. Using the dynamical response calculated in Ref. 13, in Sec. II we discuss both bulk and surface magnetostatic modes in this nonsymmetric geometry. The same geometry is used in Sec. III to discuss the behavior of the retarded modes. In Sec. IV, we carry out a 
theoretical calculation of the reflectivity for an attenuated total reflection (ATR) experiment, which can be used to study the polaritons calculated in Sec. III. Finally, in Sec. V, we summarize the main results.

\section{BULK AND SURFACE MAGNETOSTATIC MODES}

As remarked in Ref. 13, the result for the dynamic susceptibility tensor, when the external dc magnetic field is in an oblique direction, is cumbersome. On the other hand, the rich spectrum given by Almeida and Mills (Fig. 3 of Ref. 13) increases one's curiosity in how these modes are modified if the surrounding of the sample is slightly changed. We want to stress that, even if the equilibrium configuration of the magnetic moment of each sublattice is known, i.e., the canting angles $\alpha_{a}$ and $\alpha_{b}$ shown in Fig. 1 (b) are determined, it is still hard to get analytical expressions for the physical properties under investigation although it is not overly complicated for numerical results to be obtained. In this paper we investigate the behavior of the bulk and surface modes for a two-sublattice uniaxial antiferromagnet, considering the case where the symmetry of the magnetic system is broken by an external static Zeeman field applied nonparallel to the easy axis. The geometry considered here is an antiferromagnetic specimen, filling the semi-infinite half-space $y \geq 0$ with the anisotropy direction parallel to the $z$ axis while the dc external magnetic field lies in the $z x$ plane.

The magnetostatic limit is characterized by the absence of retardation terms in Maxwell's equations. This corresponds to making the time derivatives equal to zero in these equations. This fact allows us to write the demagnetizing field, generated by the spin motion (h), as the gradient of the magnetic scalar potential $\Phi_{m}$, namely,

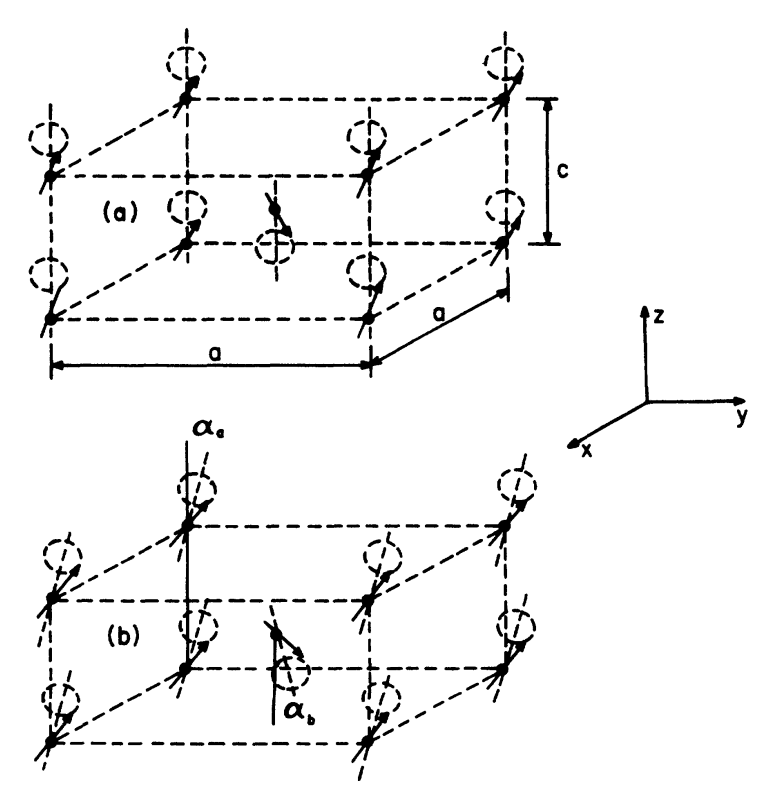

FIG. 1. A schematic illustration of the unit cell of an uniaxial antiferromagnet and the spin motion when (a) no magnetic field is present and (b) a dc external magnetic field is applied in an oblique direction. $\mathbf{h}=-\nabla \Phi_{m}$. Outside the material $(y<0), \Phi_{m}$ is a solution of Laplace's equation and its spatial dependence is given by

$$
\Phi_{m}=\Phi_{0}^{\text {out }} \exp \left(i k_{x} x+i k_{z} z+k_{\|} y\right)
$$

with $k_{\|}=\left(k_{x}^{2}+k_{z}^{2}\right)^{1 / 2}$ On the other hand, inside the material $(y>0)$, we find the scalar potential given by a plane wave with wave vector $\mathbf{k}=k\left(\cos \phi_{x}, \cos \phi_{y}, \cos \phi_{z}\right)$, with $\phi_{i}$ $(i=x, y, z)$ denoting the angles between $\mathbf{k}$ and the $x, y, z$ axis, respectively. Explicitly we have

$$
\Phi_{m}=\Phi_{0}^{\mathrm{in}} \exp \left[i k\left(x \cos \phi_{x}+y \cos \phi_{y}+z \cos \phi_{z}\right)\right] .
$$

Straightforward algebraic manipulations give the following implicit dispersion relation for bulk polaritons:

$$
\sum_{i} \mu_{i i} \cos ^{2} \phi_{i}=0, i=x, y, z
$$

Here $\mu_{i i}$ are the diagonal elements of the magnetic permeability tensor $\overleftrightarrow{\mu}=\overrightarrow{\mathrm{I}}+4 \pi \overleftrightarrow{\chi}$ with $\overleftrightarrow{\mathrm{I}}$ denoting the identity tensor and $\overleftrightarrow{\chi}$ the dynamic response of the system (dynamic susceptibility tensor) as calculated by Almeida and Mills. ${ }^{13}$

For surface modes, the solution of the magnetic scalar potential, for $y \geq 0$, is given by

$$
\Phi_{m}=\Phi_{0}^{\mathrm{in}} \exp \left[i k_{\|}(\cos \phi+\sin \phi)-\beta y\right],
$$

where $\phi$ is the angle between the wave vector of the mode and the $x$ axis and $\beta$ is a parameter which must be greater than zero. Substituting these expressions in Maxwell's equations, we find the following equations involving $\beta$

$$
\frac{\beta^{2}}{k^{2}}=\frac{1}{\mu_{y y}}\left[\mu_{x x} \sin ^{2} \phi+\mu_{z z} \cos ^{2} \phi\right]
$$

and

$$
\frac{\beta}{k}=-\frac{\left(1-\mu_{y x} \cos \phi-\mu_{y z} \sin \phi\right)}{\mu_{y y}} .
$$

The effect of the external dc field appears in the modes via the permeability tensor $\overleftrightarrow{\mu}$ since this quantity depends on the angles between the static magnetization of each sublattice and the anisotropy axis. If these angles are available, the dispersion relation for bulk and surface polaritons can be obtained through numerical analysis of the equations above. From Eqs. (2.8a) and (2.8b) of Ref. 13 , we find that these angles obey a nonlinear homogeneous system of equations given by

$$
\begin{aligned}
& \omega_{0} \sin \left(\theta-\alpha_{a}\right)-\omega_{e} \sin \left(\alpha_{a}+\alpha_{b}\right)-\frac{1}{2} \omega_{a} \sin \left(2 \alpha_{a}\right)=0, \\
& \omega_{0} \sin \left(\theta+\alpha_{b}\right)-\omega_{e} \sin \left(\alpha_{a}+\alpha_{b}\right)-\frac{1}{2} \omega_{a} \sin \left(2 \alpha_{b}\right)=0 .
\end{aligned}
$$

Here $\theta$ is the angle between the external field and the anisotropy axis. We are using the characteristic frequencies $\omega_{0}=\gamma H_{0}, \omega_{a}=\gamma H_{a}$, and $\omega_{e}=\gamma H_{e}$, and the angles $\alpha_{a}$ and $\alpha_{b}$ as defined in Fig. 1. The solutions for the non- 
linear set of equations (2.7) are plotted in Figs. 2(a) and 2(b) for $\mathrm{MnF}_{2}\left(H_{e}=550.0 \mathrm{kOe}, H_{a}=7.85 \mathrm{kOe}, M_{s}=0.6\right.$ $\mathrm{kG})$ and $\mathrm{FeF}_{2}\left(H_{e}=540.0 \mathrm{kOe}, H_{a}=200.0 \mathrm{kOe}\right.$, $\left.M_{s}=0.55 \mathrm{kG}\right)$, respectively.

With these results in hand, the task is to get the dispersion relation for the bulk and surface polaritons through numerical analysis of Eqs. (2.6) and (2.7). We show in Figs. 3(a) and 3(b) the results obtained for surface and bulk frequencies of the magnetostatic modes in $\mathrm{MnF}_{2}$ and $\mathrm{FeF}_{2}$ as a function of the propagation angle for the magnetic fields indicated in the caption. Recall that $\phi=0$ means propagation parallel to the $x$ axis while $\phi=\pi / 2$ means modes with the wave vector parallel to the anisotropy axis.

It must be observed that the unresolved crossing displayed in Fig. 3 of Ref. 13, under the conditions established here, is completely opened up and also a forbidden region for surface modes appears around the crossing point. Of course, we are dealing with conditions slightly different from those in Ref. 13 and the modifications are more drastic if either the strength of the dc field is increased or the value of $\theta$ is decreased.
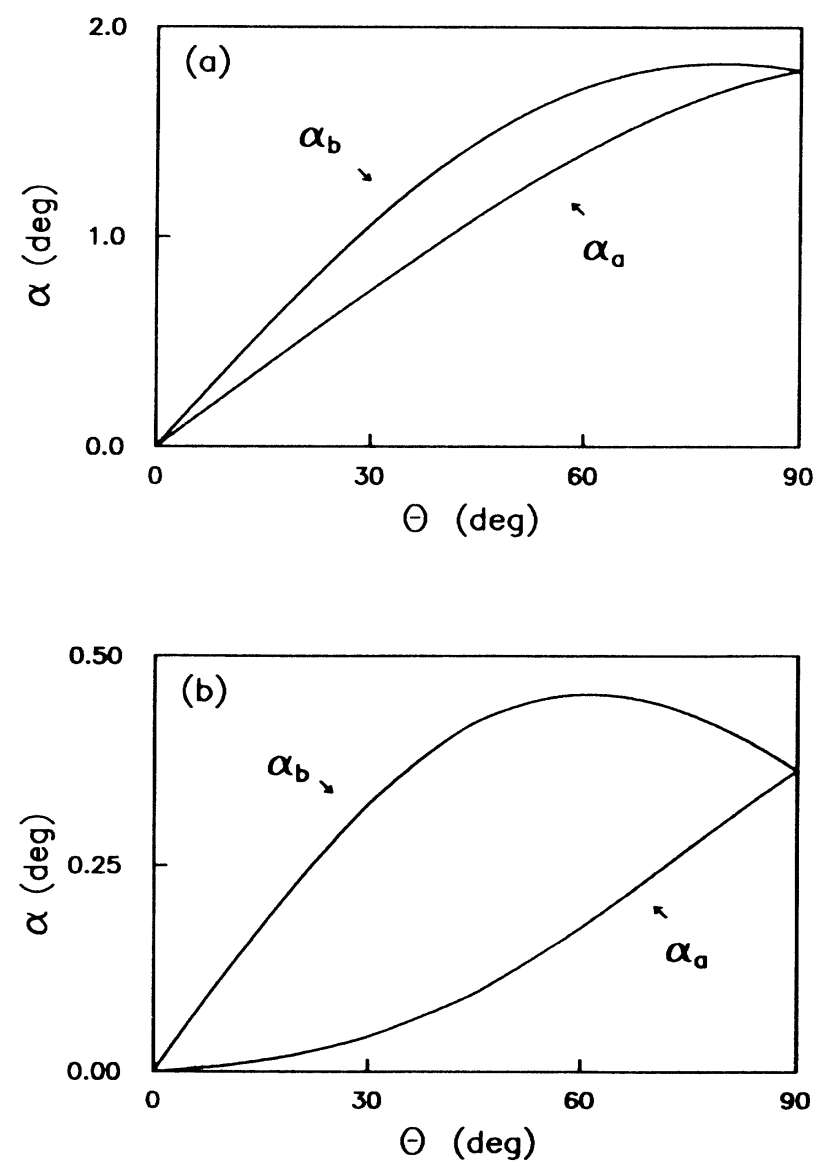

FIG. 2. Behavior of angles $\alpha_{a}$ and $\alpha_{b}$ (as defined in Fig. 1) with the angle $\theta$ between the Zeeman field $H_{0}$ and the easy axis for (a) $\mathrm{MnF}_{2}, H_{0}=7.0 \mathrm{kOe}$, and (b) $\mathrm{FeF}_{2}, H_{0}=40.0 \mathrm{kOe}$.
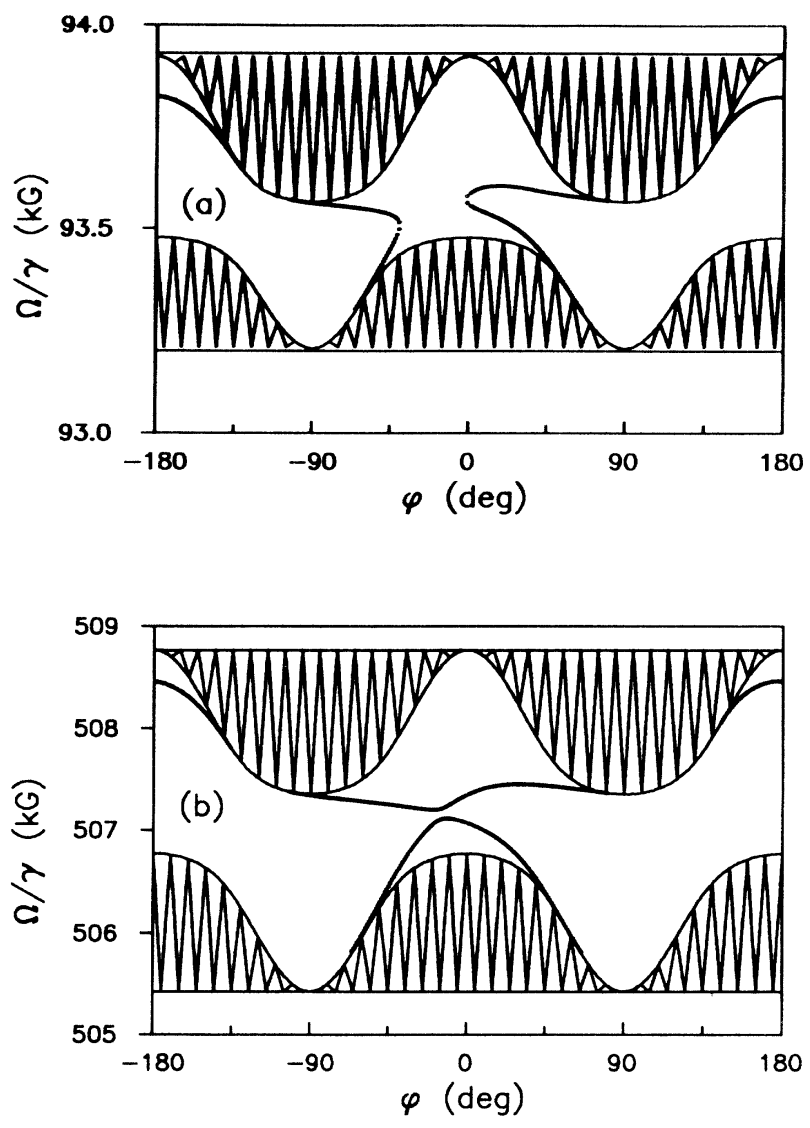

FIG. 3. Surface (solid line) and bulk (shaded area) magnetostatic modes for various values of the propagation angle $\varphi$ for (a) $\mathrm{MnF}_{2}, H_{0}=7.0 \mathrm{kOe}$, and $\theta=89^{\circ}$; and (b) $\mathrm{FeF}_{2}, H_{0}=40.0 \mathrm{kOe}$ and $\theta=89^{\circ}$.

\section{RETARDED SURFACE MODES}

In this section we study the surface polaritons on a semi-infinite uniaxial antiferromagnetic sample under the conditions described in Sec. II. In this case we have to keep the time derivatives in Maxwell's equations and define the polarization of the modes under investigation. We choose the TE modes (with the electric field perpendicular to the incident plane) and the same geometry used in the last section to write the electric field $\mathbf{E}$ and the induction magnetic field $\mathbf{B}=\overleftrightarrow{\mu} \cdot \mathbf{H}$ as

$$
\begin{aligned}
& \mathbf{E}=\left(E_{x} \hat{\mathbf{x}}+E_{z} \hat{\mathbf{z}}\right) \exp [i(\mathbf{k} \cdot \mathbf{r}-\omega t)], \\
& \mathbf{B}=\left(B_{x} \hat{\mathbf{x}}+B_{y} \hat{\mathbf{y}}+B_{z} \hat{z}\right) \exp [i(\mathbf{k} \cdot \mathbf{r}-\omega t)],
\end{aligned}
$$

with $\mathbf{k}$ and $\omega$ denoting the wave vector and frequency of the polariton. Using these definitions in Maxwell's equations, we find

$$
\boldsymbol{\nabla} \times\left[\overleftrightarrow{\boldsymbol{\epsilon}}^{-1} \cdot(\boldsymbol{\nabla} \times \mathbf{H})\right]+\frac{\omega}{c^{2}} \overleftrightarrow{\mu} \cdot \mathbf{H}=\mathbf{0}
$$

and

$\boldsymbol{\nabla} \cdot(\overleftrightarrow{\mu} \cdot \mathbf{h})=0$ 
with the magnetic field $\mathbf{H}$ defined as

$$
\mathbf{H}^{\text {out }}=\left(H_{x}, H_{y}^{\text {out }}, H_{z}\right) \exp \left[i\left(\mathbf{k}_{\|} \cdot \mathbf{r}-\omega t\right)+\alpha y\right] \text { if } y \leq 0
$$

and

$$
\mathbf{H}^{\mathrm{in}}=\left(H_{x}, H_{y}^{\mathrm{in}}, H_{z}\right) \exp \left[i\left(\mathbf{k}_{\|} \cdot \mathbf{r}-\omega t\right)-\beta y\right] \text { if } y \geq 0,
$$

where the parameters $\alpha$ and $\beta$ must be greater than zero. Using these expressions for the magnetic field in (3.2) combined with the usual boundary conditions, we find the following homogeneous systems of equations:

$$
\begin{gathered}
{\left[\boldsymbol{M}_{1}\left(\beta \mu_{y x}-i k_{\|} \boldsymbol{M}_{2}\right)-\left(i k_{\|} \boldsymbol{M}_{4}-\beta \mu_{y y}\right)\left(i k_{\|} \beta c^{2} \cos \phi+\omega^{2} \epsilon_{1} \mu_{y x}\right)\right] H_{x}} \\
+\left[\boldsymbol{M}_{1}\left(\beta \mu_{y z}-i k_{\|} \boldsymbol{M}_{3}\right)-\left(i k_{\|} \boldsymbol{M}_{4}-\beta \mu_{y y}\right)\left(i k_{\|} \beta c^{2} \sin \phi+\omega^{2} \epsilon_{1} \mu_{y z}\right)\right] H_{z}=0, \\
{\left[\boldsymbol{M}_{1}\left(i k_{\|} \cos \phi+\alpha \mu_{y x}\right)-\alpha \mu_{y y}\left(\omega^{2} \epsilon_{1} \mu_{y x}+i k_{\|} \beta c^{2} \cos \phi\right)\right] H_{x}} \\
+\left[M_{1}\left(i k_{\|} \sin \phi+\alpha \mu_{y z}\right)-\alpha \mu_{y y}\left(\omega^{2} \epsilon_{1} \mu_{y z}+i k_{\|} \beta c^{2} \cos \phi\right)\right] H_{z}=0,
\end{gathered}
$$

and

$$
\begin{gathered}
{\left[\left(i k_{\|} \beta c^{2} \cos \phi+\omega^{2} \epsilon_{1} \mu_{x y}\right)\left(i k_{\|} \beta c^{2} \cos \phi+\omega^{2} \epsilon_{1} \mu_{y x}\right)+M_{1}\left(k_{\|}^{2} c^{2} \sin ^{2} \phi-\beta^{2} c^{2}-\omega^{2} \epsilon_{1} \mu_{x x}\right)\right] H_{x}} \\
+\left[\left(i k_{\|} \beta c^{2} \cos \phi+\omega^{2} \epsilon_{1} \mu_{x y}\right)\left(i k_{\|} \beta c^{2} \sin \phi+\omega^{2} \epsilon_{1} \mu_{y z}\right)-M_{1}\left(k_{\|}^{2} c^{2} \cos \phi \sin \phi+\omega^{2} \epsilon_{1} \mu_{x z}\right)\right] H_{z}=0, \\
{\left[\left(i k_{\|} \beta c^{2} \sin \phi+\omega^{2} \epsilon_{1} \mu_{z y}\right)\left(i k_{\|} \beta c^{2} \cos \phi+\omega^{2} \epsilon_{1} \mu_{y x}\right)-M_{1}\left(k_{\|}^{2} c^{2} \sin \phi \cos \phi+\omega^{2} \epsilon_{1} \mu_{z x}\right)\right] H_{x}} \\
+\left[\left(i k_{\|} \beta c^{2} \sin \phi+\omega^{2} \epsilon_{1} \mu_{z y}\right)\left(i k_{\|} \beta c^{2} \sin \phi+\omega^{2} \epsilon_{1} \mu_{y z}\right)+M_{1}\left(k_{\|}^{2} c^{2} \cos ^{2} \phi+\beta^{2} c^{2}+\omega^{2} \epsilon_{1} \mu_{z z}\right)\right] H_{z}=0,
\end{gathered}
$$

where $\quad M_{1}=\left(\omega^{2} \epsilon_{1} \mu_{y y}-k_{\|}^{2} c^{2}\right), \quad M_{2}=\mu_{x x} \cos \phi+\mu_{z x} \sin \phi$, $M_{3}=\mu_{x z} \cos \phi+\mu_{z z} \sin \phi$, and $M_{4}=\mu_{x y} \cos \phi+\mu_{z y} \sin \phi$.

The condition for nontrivial solutions of the homogeneous systems of Eqs. (3.4) and (3.5) provides two homogeneous polynomials in the $\beta$ variable, with coefficients depending on $k_{\|}$and $\omega$. The simultaneous solution of these polynomials gives the dispersion relation, provided $\beta \geq 0$. We numerically solve these equations taking $\theta=\pi / 2$ and the results for the dispersion relation for polaritons propagating in arbitrary directions

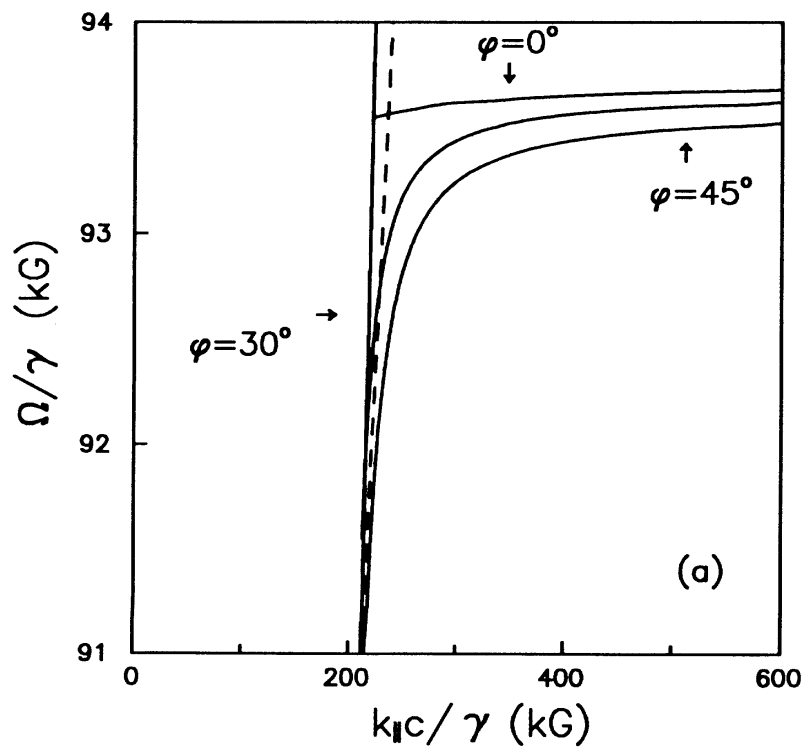

relative to the $x$ axis is shown in Figs. 4(a) and 4(b) for $\mathrm{MnF}_{2}$ and $\mathrm{FeF}_{2}$, respectively. It should be observed, not surprisingly, that the frequency of the polariton with large value of the wave vector is exactly the same as the magnetostatic mode (calculated in Sec. II) propagating in the same direction. It should also be remarked that the modes which have their directions of propagation close to the $x$ axis have their dispersion relation very flat. This fact allow us to conclude that retarded terms in Maxwell's equations have a very small influence (or even

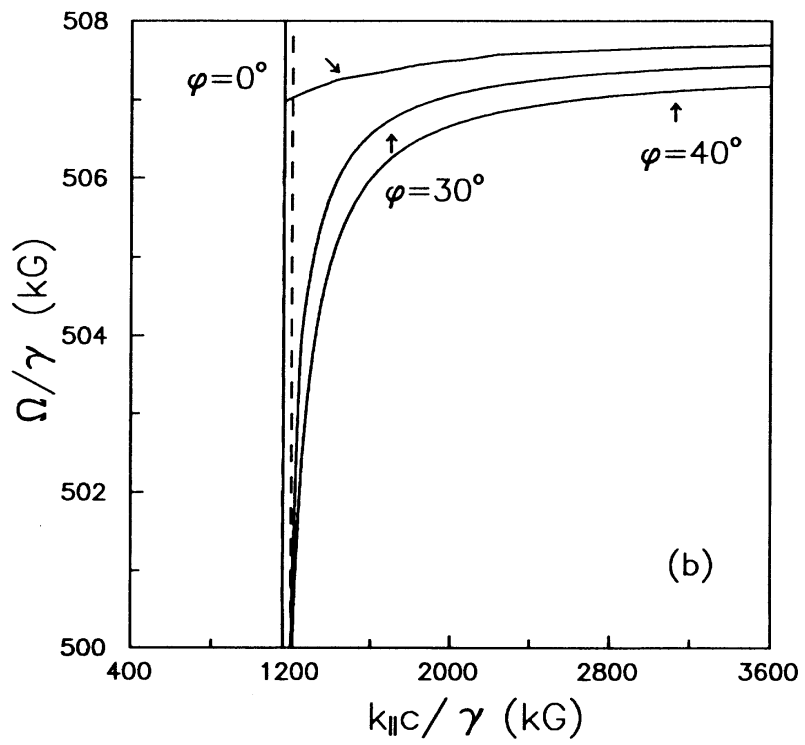

FIG. 4. Dispersion relation curves of retarded modes for $\theta=\pi / 2$ and the angle $\varphi$ between the wave vector and $x$ axis indicated for (a) $\mathrm{MnF}_{2}, H_{0}=7.0 \mathrm{kOe}$, and (b) $\mathrm{FeF}_{2}, H_{0}=40.0 \mathrm{kOe}$. The dashed curve is the scan line used in the ATR spectrum calculation. 
none) on the polaritons propagating perpendicular to the easy axis. Furthermore, their frequencies are almost independent of the orientation of the dc external field.

\section{ATTENUATED TOTAL REFLECTION}

One of the experimental techniques used to study retarded polaritons is the so-called attenuated total reflection spectroscopy. ${ }^{14}$ This technique is described elsewhere and we chose the geometry shown in Fig. 5 to calculate the spectrum which should be observed in this experiment. We assume that the radiation of frequency $\omega$ is incident at an angle $\theta_{i}$ in a semicircular prism of dielectric constant $\epsilon_{s}$, with $\theta_{i}>\theta_{c}$, where $\theta_{c}$ is the critical angle for total internal reflection. This prism is separated from the specimen by an air gap of thickness $d$. Therefore, polaritons with wave vector $k=\sqrt{\epsilon_{s}}(\omega / c) \sin \theta_{i}$ and frequency $\omega$ (if any exists) will be excited and a dip must appear in the reflectivity. The expression for the reflection coefficient $R$, defined as the ratio of the reflected to incident energy, is obtained from a straightforward calculation and so we just quote the result:

$$
R=\frac{\left|t_{1}-t_{2}\right|^{2}}{\left|t_{1}+t_{2}\right|^{2}}
$$

with $t_{1}$ and $t_{2}$ given by

$$
t_{1}=\left(1+\frac{\left(\alpha \mu_{x x}-\beta\right)}{\left(\alpha \mu_{x x}+\beta\right)} \exp (2 \alpha d)\right)
$$

and

$$
t_{2}=-i \frac{\alpha}{k_{\perp}}\left[1-\frac{\left(\alpha \mu_{x x}-\beta\right)}{\left(\alpha \mu_{x x}+\beta\right)} \exp (2 \alpha d)\right],
$$

where

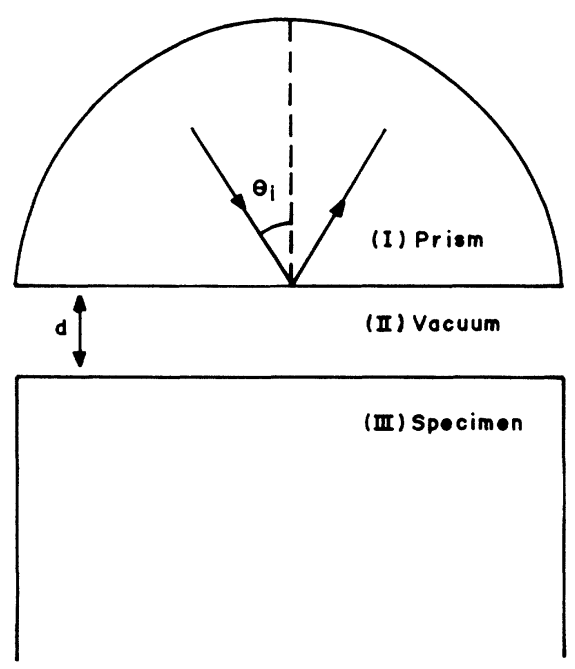

FIG. 5. Geometry used to calculate the ATR spectrum.

$$
\begin{aligned}
& \alpha=1 / c \sqrt{k_{\|}^{2} c^{2}-\omega^{2}}, \\
& \beta=1 / c \sqrt{\left(k_{\|}^{2} c^{2} / \mu_{y y}-\omega^{2}\right) \mu_{x x}} .
\end{aligned}
$$

Figures 6(a) and 6(b) show the behavior of $R$ when, for a fixed value of the incident angle, the frequency of the incident radiation is scanned from the extreme values indicated in the top horizontal axis and under the conditions described in the captions. We have used the physical parameters for $\mathrm{MnF}_{2}$ and $\mathrm{FeF}_{2}, \epsilon_{s}=10.0$ (Si prism) and the values of the thickness $d$ indicated in the figures, to get the numerical results for the reflection coefficient. From these results we can see that the dips of Figs. 6(a) and $6(\mathrm{~b})$ correspond to the intersection of the scan lines of the Figs. 4 (slashed line) with the dispersion curves. We have very sharp dips because we have used a small value for the damping factor (included in $\overleftrightarrow{\mu}$ via the replacement of $\omega$ by $\omega+i \delta, \delta \simeq 150.0 \mathrm{G}$ ).
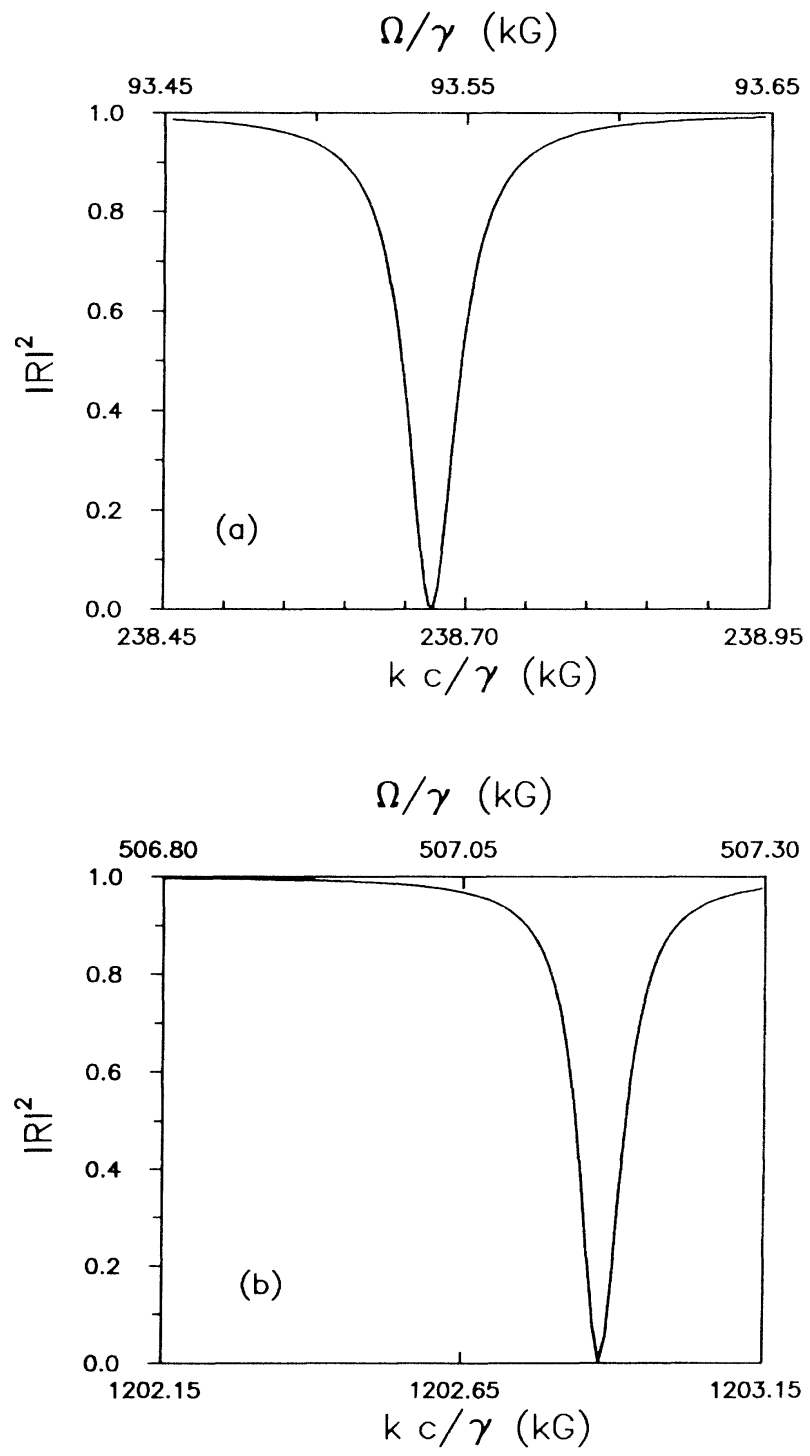

FIG. 6. ATR spectra obtained with the geometry shown in Fig. 5 and following the scan line of Figs. 4(a) (a) and 4(b) (b). 


\section{CONCLUSION}

In summary, for a uniaxial Heisenberg antiferromagnetic material, we have done a complete theoretical investigation of the influence of an external dc magnetic field applied in arbitrary direction relative to its easy axis on the polariton dispersion relations. We would like to remark that there are qualitative as well as quantitative effects on the polariton spectrum resulting from changes in the magnetic properties of the surroundings. It is clear that these "magnetic field controlled polaritons" are very sensitive to the strength of the static field as well as to the orientation of this field relative to the $c$ axis. Of course, these effects are more evident if a higher external magnetic field is applied or if a softer magnetic material, like a Y-Gd superlattice, is used. It was shown recently ${ }^{15}$ that
Y-Gd can be described by a permeability tensor which has the same qualitative behavior as the one used in the present calculation. Nevertheless, the intrinsic characteristic of this material allows it to be much more sensitive to the presence of an oblique magnetic field. A small magnetic field (few G) gives to $\alpha_{a}$ and $\alpha_{b}$ larger values inducing much higher nonsymmetry than that studied here. The ATR spectrum, also calculated here, suggests that this technique is a variable experimental method capable of detecting these magnetic polaritons.

\section{ACKNOWLEDGMENTS}

This work was partially supported by the Brazilian Agency CNPq. One of us (TLF) also thanks the Brazilian Agency CAPES for financial support.
*Present address: Department of Physics, University of Colorado at Colorado Springs, Colorado Springs, Colorado 809077150 .

${ }^{1}$ R. Damon and J. Eshbach, J. Phys. Chem. Solids 19, 308 (1961).

${ }^{2}$ R. Loudon and P. Pincus, Phys. Rev. 132, 673 (1963).

3J. P. Kotthaus and V. Jaccarino, Phys. Lett. 28, 1649 (1972).

${ }^{4}$ D. L. Mills and E. Burstein, Rep. Prog. Phys. 37, 817 (1974).

${ }^{5}$ R. E. Scott and D. L. Mills, Phys. Rev. B 15, 3545 (1977).

${ }^{6}$ P. Grünberg and F. Metawe, Phys. Rev. Lett. 39, 1561 (1977).

${ }^{7}$ R. E. Camley and D. L. Mills, Phys. Rev. B 18, 4821 (1978).

${ }^{8}$ R. E. Camley, Phys. Rev. Lett. 45, 283 (1980).
${ }^{9}$ R. E. Camley and D. L. Mills, Phys. Rev. B 26, 1280 (1982).

${ }^{10}$ B. Lüthi, D. L. Mills, and R. E. Camley, Phys. Rev. B 28, 1475 (1983).

${ }^{11}$ R. E. Camley, Surf. Sci. Rep. 7, 103 (1987), and references therein.

${ }^{12}$ R. M. Toussaint, D. W. Hone, V. Jaccarino, and S. M. Rezende, Phys. Rev. B 30, 3859 (1984).

${ }^{13}$ N. S. Almeida and D. L. Mills, Phys. Rev. B 37, 3400 (1988).

${ }^{14}$ N. S. Almeida and D. R. Tilley, Solid State Commun. 73, 23 (1990).

${ }^{15}$ N. S. Almeida and D. L. Mills, Phys. Rev. B 38, 6698 (1988); 39, 12 339(E) (1989). 